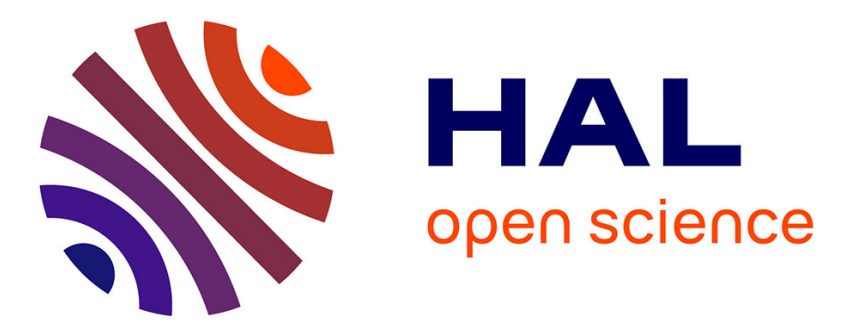

\title{
Predictive control over wireless multi-hop networks
}

Emmanuel Witrant, Pan Gun Park, Mikael Johansson, Carlo Fischione, Karl

Henrik Johansson

\section{To cite this version:}

Emmanuel Witrant, Pan Gun Park, Mikael Johansson, Carlo Fischione, Karl Henrik Johansson. Predictive control over wireless multi-hop networks. CCA 2007 - 16th IEEE International Conference on Control Applications, Oct 2007, Singapour, Singapore. hal-00196875

\section{HAL Id: hal-00196875 https://hal.science/hal-00196875}

Submitted on 13 Dec 2007

HAL is a multi-disciplinary open access archive for the deposit and dissemination of scientific research documents, whether they are published or not. The documents may come from teaching and research institutions in France or abroad, or from public or private research centers.
L'archive ouverte pluridisciplinaire HAL, est destinée au dépôt et à la diffusion de documents scientifiques de niveau recherche, publiés ou non, émanant des établissements d'enseignement et de recherche français ou étrangers, des laboratoires publics ou privés. 


\title{
Predictive control over wireless multi-hop networks
}

\author{
Emmanuel Witrant, Pan Gun Park, Mikael Johansson, Carlo Fischione and Karl Henrik Johansson*
}

\begin{abstract}
Remote control over wireless multi-hop networks is considered. Time-varying delays for the transmission of sensor and control data over the wireless network are caused by a randomized multi-hop routing protocol. The characterstics of the routing protocol together with lower-layer network mechanisms give rise to a delay process with high variance and stepwise changing mean. A new predictive control scheme with a delay estimator is proposed in the paper. The estimator is based on a Kalman filter with a change detection algorithm. It is able to track the delay mean changes but efficiently attenuate the high frequency jitter. The control scheme is analyzed and its implementation detailed. Network data from an experimental setup are used to illustrate the efficiency of the approach.
\end{abstract}

\section{Introduction}

There are major advantages in terms of increased productivity and reduced installation costs in the use of wireless communication technology in industrial control systems. Several wireless technologies are currently reaching the plant and workshop floor addressing a range of applications. A multi-hop wireless network is one such technology that offers great flexibility, but also challenges when it comes to predictability. These networks are used in emerging applications in industrial automation and dedicated algorithms, such as randomized multi-hop routing protocols [1], were recently shown to be useful in these applications. From the perspective of designing control systems utilizing wireless multi-hop networks, one of the most significant characteristics is the need of hiding the system complexity through suitable abstraction of the networks and their

${ }^{*}$ The authors are with KTH, Osquldas väg 10, SE-100 44 Stockholm (Sweden). The corresponding author email is emmanuel.witrant@ee.kth.se.

This work has been supported in part by the European Commission through the projects SOCRADES and HYCON, the Swedish Agency for Innovation Systems via the Nordite project VISA, the Swedish Research Council and the Swedish Foundation for Strategic Research. nodes. One simple but important way of doing this is by interpreting the network as a communication channel with time-varying delay.

Experimental results on telerobotics over TCP/IP networks illustrate the importance of varying transmission delays in the control loop [2], where the authors combine a generic estimation/prediction control scheme with a buffering of the received packets, which compensates for the delays jitter. While the buffer may decrease the transient performances of the closed-loop system [3], this work motivates the use of estimation/prediction schemes to achieve robust and efficient control over networks. Another interesting experimental result is provided in [4], where a peer-to-peer wireless network (802.11b) and a UDP protocol are used to transmit the sensors and actuators signals.

The main contribution of the paper is a new scheme for control over wireless multi-hop networks. The characteristics of the routing protocol together with lowerlayer network mechanisms give rise to a delay process with high variance and stepwise changing mean, which is particularly difficult to model. Here we use a loworder model proposed in [5] and propose a new predictive control scheme with a delay estimator. The estimator is based on a Kalman filter with a change detection algorithm [5]. It is able to track the delay mean changes but efficiently attenuate the high frequency jitter. Compared to similar works such as [6], where an output feedback scheme uses the maximal value of the delay and GPS synchronization for remote control over Internet, we explicitly compensate for the time-varying delay effect. Network data from an experimental setup are used to illustrate the efficiency of the approach.

The outline of the paper is as follows. Section II defines the considered problem of control over a wireless multi-hop network. The wireless network and its randomized routing protocol is described in Section III. The time-delay estimator is presented in Section IV. Section V presents the controller and its delay compensator. Experimental results are given in Section VI followed by the conclusions in Section VII. 


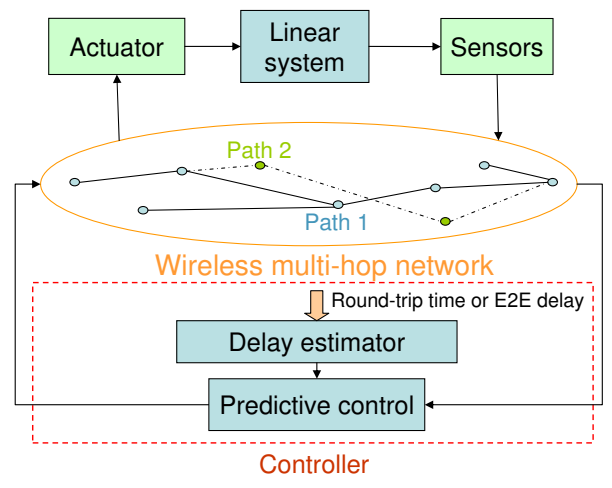

Figure 1. Overview of the system and control setup.

\section{Problem description}

The problem considered is depicted in Fig. 1, where a linear system is remotely controlled over a wireless multi-hop network. We suppose that round-trip time or end-to-end delay measurements (i.e., between the sensors and controller, which also implies a proper clock synchronization) are available. A predictive control law uses the filtered measurements to compensate for variations induced by the multi-hop network. More precisely, we consider the remote control of linear systems that write as

$$
\begin{aligned}
& \dot{x}(t)=A x(t)+B v(t), \quad x(0)=x_{0} \\
& y(t)=C x(t)
\end{aligned}
$$

where $x \in R^{n}$ is the internal state, $v \in R$ is the system input, $y \in R^{m}$ is the system output, and $A, B, C$ are matrices of appropriate dimensions. The pairs $(A, B)$ and $(A, C)$ are assumed to be controllable and observable, respectively, but no assumption is made on the stability of $A$. The delays introduced by the network dynamics are defined as

- $\tau_{1}(t)$ : delay between the sensors and the controller,

- $\tau_{2}(t)$ : delay between the controller and the actuator.

The actuator is event-driven and uses the last received signal. Packet losses influence the actuation as

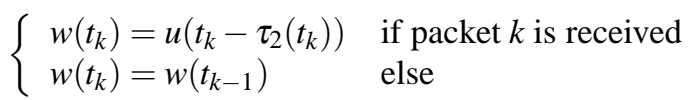

where $w(\cdot)$ is the signal received by the actuator from the network, $u(\cdot)$ is the controller output and $v(t)=$ $w\left(t_{k}\right) \forall t \in\left[t_{k}, t_{k+1}\right)$. The chronology of the signals is indicated in the transmitted data in order to reconstruct their history on the receiver site. The receivers consider only the most recent information, meaning that a packet is also considered as lost if a more recent one is received first.

\section{Wireless multi-hop networks}

In many scenarios of relevant interest, wireless multi-hop networks do not have fixed communication paths, but the end-to-end path followed by packets happens according to a dynamic selection of hops $[7,8]$. A major technique to ensure power savings and longer network lifetime is to turn off a node whenever its presence is not strictly required for the correct operation of the network $[8,9,1]$, which means that the network topology is changing randomly.

Consider the multi-hop network reported in Fig. 1, where the transmitter to receiver path is composed of a time-varying number of hops $h(t)$. For each node, denoted by $i=1 \ldots h(t)$, we can consider the following sources of delay:

- the time $\alpha_{i}$ to wait before sending a data packet, which is typically a random variable;

- the time $F$ to forward a data packet once the connection with the next node is set up, which is constant and contains the propagation and transmission delays;

- possibly the time $\beta_{i}$ induced by an Automatic Repeat reQuest (ARQ) mechanism, which retransmits a packet for a predefined number of time if no acknowledgement is received.

The end-to-end delay can then be expressed as follows:

$$
\tau(t)=h(t) F+\sum_{i=1}^{h(t)}\left(\alpha_{i}+\beta_{i}\right)
$$

If one looks at the outcomes of (4), there are three possible behaviors. The low frequency delays are due to changes in the number of hops $h(t)$. The middle frequency ones depend on the selection of the next awaken hop for a fixed number of clusters (related to $\mu_{c, i}$ in the example considered), and the high frequency behavior is related to the transmissions between nodes.

\section{Time-delay estimator}

A critical component of the proposed control strategy is the delay estimator, whose output is used to set the time-varying horizon of the predictive controller. Examining the real delay traces from our network, we 
see that the delay exhibits a large variability and persistent changes in the mean. Although these changes can be traced back to a particular feature of our protocol (discussed in the previous section) similar effects have been reported for a wide range of network quantities and network technologies [10]. Since the controlleractuator latency is not immediately available to the controller, but has to be deduced from the (delayed information about the) controller-actuator round-trip time, we will look for a delay estimator that allows to smooth out the fast variations while being able to react quickly to the changes in mean.

To this end, we propose to use a combination of Kalman filter and CUMSUM change-detection (cf., [5, 11]). The underlying signal model is

$$
\begin{aligned}
\tau_{k+1} & =\tau_{k}+v_{k} \\
r_{k} & =c \tau_{k}+w_{k}
\end{aligned}
$$

where $\tau_{k}$ is the one-way latency, $r_{k}$ is the measured time, $c$ is the number of delays included in $r_{k}$ (i.e., 1 if the end-to-end delay of the channel is measured and 2 if it is the round trip time), $w_{k}$ is a white-noise sequence with covariance $R_{k}$ and

$v_{k}= \begin{cases}0 & \text { with probability } 1-q, \\ v & \text { with probability } q, \text { where } \operatorname{Cov}\left(v_{k}\right)=q^{-1} Q_{k}\end{cases}$

models the abrupt changes in the mean. The associated Kalman filter updates are given by

$$
\begin{aligned}
\hat{\tau}_{k+1} & =\hat{\tau}_{k}+K_{k}\left(r_{k}-c \hat{\tau}_{k}\right) \\
K_{k} & =\frac{c P_{k-1}}{R_{k}+c^{2} P_{k-1}} \\
P_{k} & =P_{k-1}-\frac{c^{2} P_{k-1}^{2}}{R_{k}+c^{2} P_{k-1}}+Q_{k}
\end{aligned}
$$

The tracking ability of the filter is proportional to the variance of the process noise $Q_{k}$, but increasing $Q_{k}$ also decreases the smoothing effect that we are looking for.

To improve performance, we run a CUMSUM detector in parallel with the Kalman filter. The detector looks at the prediction errors $\varepsilon_{k}=r_{k}-c \hat{\tau}_{k}$, sums up the negative and positive prediction errors

$$
\begin{aligned}
& g_{k}^{+}=\max \left(g_{k-1}+\varepsilon_{k}-\kappa, 0\right) \\
& g_{k}^{-}=\max \left(g_{k-1}-\varepsilon_{k}-\kappa, 0\right)
\end{aligned}
$$

and monitors if $g_{k}^{+}$or $g_{k}^{-}$exceeds a threshold $g_{t h r}$. Here, the parameter $\kappa$ is a design parameter that specifies the negative drift (see [11] for design guidelines). At these threshold crossings, $Q_{k}$ is set to a large value, $g^{+}$and $g^{-}$are reset to zero, $\hat{\tau}_{k}$ is set to $c^{-1} r_{k}$ and the Kalman filter iterations resumed. The combined Kalman-filter and CUMSUM test allows to achieve both fast tracking of the changes in the mean and good suppression of the high-frequency variations.

\section{Predictive control approach}

The control approach proposed here is based on the state predictor with a time-varying horizon first proposed in [12]. The main advantage of such method compared to other delay compensation strategies [13] is to allow for a finite spectrum assignment on systems with time-varying delays. An overview on the use of state predictors in networked control systems is provided in [3], where it is compared with more classical control approaches.

\subsection{Ideal delay compensation}

We use the results established in [14] and subsequent works, where the delay induced by the network dynamics is explicitly taken into account in the control setup. For simplicity and to focus on the network effect on the closed-loop system, we suppose that the full delayed state ${ }^{1} x\left(t-\tau_{1}\right)$ is available to set the control law. When there is no packet loss and the delay is fully known (and can be predicted), the main theorem in [14] can be extended to the case where both communication channels experience a delay as follows.

Theorem 5.1 Consider the system

$$
\dot{x}(t)=A x(t)+B u\left(t-\tau_{2}\right), \quad x(0)=x_{0}
$$

with $(A, B)$ a controllable pair. Assume that the network dynamics is such that the following holds for $\tau_{1}$ and $\tau_{2}$

A1) $\tau_{1,2} \in \mathscr{C}\left(R^{+},\left[0, \tau_{\max }\right]\right) \quad \forall t$,

A2) $\dot{\tau}_{1,2}<1 \quad \forall t \geq t_{0}$.

Then the feedback control law

$$
\begin{aligned}
u(t)= & -K e^{A \delta}\left[e^{A \tau_{1}} x\left(t-\tau_{1}\right)\right. \\
& \left.+e^{A t} \int_{t-\tau_{1}}^{t+\delta} e^{-A \theta} B u\left(\theta-\tau_{2}(\theta)\right) d \theta\right] \\
\dot{\delta}(t)= & -\frac{\lambda}{1-d \tau_{2}(\zeta) / d \zeta} \delta+\frac{d \tau_{2}(\zeta) / d \zeta+\lambda \tau_{2}(\zeta)}{1-d \tau_{2}(\zeta) / d \zeta}
\end{aligned}
$$

with $\zeta=t+\delta, \lambda$ a positive constant, $\delta(0)=\delta_{0}$, ensures that the system trajectories $x(t)$ converge exponentially to zero.

\footnotetext{
${ }^{1}$ The time dependency of the variables $\delta(t), \tau_{1}(t)$ and $\tau_{2}(t)$ are omitted in the notations from this point.
} 
If A1-A2 hold (which correspond to a lossless network description), then the closed-loop behavior can be inferred from the time-shifted dynamics $d x / d \zeta=(A-$ $B K) x(\zeta)$ and its spectrum coincides with the spectrum of the matrix $A-|(1+\dot{\delta}) /(\dot{\tau}(t+\delta))| e^{-A \delta} B K$, where $(\cdot)$ denotes the differentiation with respect to time $t$. Considering the worst-case delay properties, the feedback gain has to be chosen such that the matrix

$$
A-\left|\frac{1}{1-\dot{\tau}_{\max }}\right| e^{-A \tau_{\max } B K}
$$

is Hurwitz.

\subsection{Use of the delay estimator}

We now consider that the control law is set using the delay estimator described in section 4. Both delays $\tau_{1}$ and $\tau_{2}$ are then replaced by $\hat{\tau}$ in the control setup description introduced in Theorem 5.1. This is motivated by the fact that the predictor architecture in used to compensate for the time-variations of the latency (i.e., the slow and medium dynamics of the delay), which are estimated with the CUMSUM Kalman filter. It is reasonable to suppose that the average values of the delays in both communication channels are close and provided by the estimator. The control law is then given as

$$
\begin{aligned}
u(t)= & -K e^{A \delta}\left[e^{A \hat{\tau}} x\left(t-\tau_{1}\right)\right. \\
& \left.+e^{A t} \int_{t-\hat{\tau}}^{t+\delta} e^{-A \theta} B u(\theta-\hat{\tau}(\theta)) d \theta\right] .
\end{aligned}
$$

The predictor horizon is computed using the actual values of the estimated delay and delay variation directly instead of the predicted ones (at time $\zeta$ ). We then approximate $\delta$ with $\hat{\delta}$, which dynamics is given by

$$
\dot{\hat{\boldsymbol{\delta}}}(t)=\frac{\dot{\hat{\tau}}(t)+\lambda(\hat{\tau}(t)-\hat{\boldsymbol{\delta}}(t))}{1-\dot{\hat{\tau}}(t)} .
$$

\subsection{Control algorithm}

Special care has to be taken in the computation of the control law (6)-(7), especially concerning the integral term used in the predictor's part. Indeed, the discretization of the integral may introduce some numerical instabilities [15]. The effect of these instabilities is further increased if the communication channel experiences packet losses and bandwidth limitation. A solution to this problem is to include the discretized integral in a dynamic control law, as described in this section.
Considering the integral term

$$
\mathscr{I}(t) \doteq e^{A t} \int_{t-\hat{\tau}}^{t+\hat{\delta}} e^{-A \theta} B u(\theta-\hat{\tau}(\theta)) d \theta
$$

computed at the time instant denoted by $k$, we have that

$$
\mathscr{I}_{k}=e^{A \hat{\tau}_{k}} \int_{0}^{\hat{\tau}_{k}+\hat{\delta}_{k}} f(\mu) d \mu
$$

with $f(\mu)=e^{-A \mu} B u\left(\min \left(\mu+t_{k}-\hat{\tau}_{k}-\hat{\tau}\left(\min \left(\mu+t_{k}-\right.\right.\right.\right.$ $\left.\left.\left.\left.\hat{\tau}_{k}, t_{k}\right)\right), t_{k}\right)\right)$ to ensure causality. $\mathscr{I}_{k}$ is discretized using the results proposed in [15], with a trapezoidal rule and included into the dynamic controller

$$
\left\{\begin{aligned}
\dot{z}(t)= & -a z(t)+b K e^{A\left(\hat{\delta}_{k}+\hat{\tau}_{k}\right)}\left[x\left(t-\tau_{1}\right)\right. \\
& \left.+\frac{t_{s}}{2} \sum_{i=1}^{n_{k}} f\left((i-1) t_{s}\right)+f\left(i t_{s}\right)\right], \\
u_{k}= & z\left(t_{k}\right)
\end{aligned}\right.
$$

where $n_{k} \doteq\left(\hat{\delta}_{k}+\hat{\tau}_{k}\right) / t_{s}, a$ and $b$ are some positive design parameters, $t_{s}$ is the sampling time set by the controller clock and $n_{k}$ is the number of steps used to discretize the integral, which is time-varying. Note that we chose the controller sampling time as the integrand step, since it sets the buffering of the past estimated delays and controller outputs. An upper bound $\dot{\bar{\tau}}_{t h r}$ is set on the maximum estimated delay variation and the dynamics of the predictor horizon is set with

$$
\dot{\hat{\delta}}(t)=\frac{\dot{\bar{\tau}}_{k}+\lambda\left(\hat{\tau}_{k}-\hat{\delta}(t)\right)}{1-\dot{\bar{\tau}}_{k}},
$$

where $\dot{\bar{\tau}}_{k}=\left(\hat{\tau}_{k}-\hat{\tau}_{k-1}\right) / t_{s}$ if $\dot{\bar{\tau}}<\dot{\bar{\tau}}_{t h r}$, and $\dot{\bar{\tau}}_{k}=\dot{\bar{\tau}}_{t h r}$ else. The issues considered in this section are summarized with the following result.

Result 5.1 Using the delay estimation provided by the CUMSUM Kalman filter described in section 4, the proposed predictive control approach is set with the dynamic controller (8), where the predictor horizon is set by the dynamics (9) and the controller gain is such that the closed-loop matrix (5) is Hurwitz.

\section{Experimental and simulation results}

In this section, the network setup is presented along with some experimental results, which are used to estimate the efficiency of the proposed control setup using appropriate simulation tools. More details on the experimental setup and protocol specifications can be found in [16]. We use the Randomized Protocol (RP) proposed in [1] and briefly discussed in Section 3. 


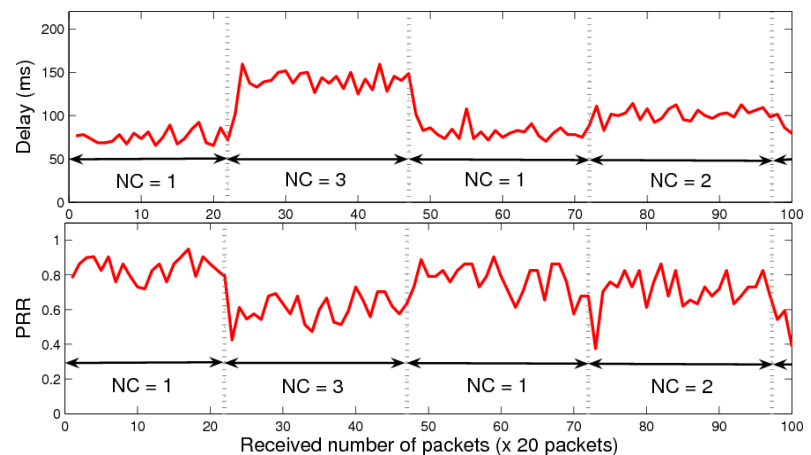

Figure 2. Average end-to-end network-induced delays (top) and packet reception rate

\subsection{Network and experimental measurements}

Our experiment is based on the RP using T-motes Sky nodes equipped with the radio controller Chipcon CC2420 $(2.4 \mathrm{GHz})$ and satisfying the communication standard IEEE 802.15.4 [17]. The source is located at $30 \mathrm{~m}$ from the destination node connected to the PC and generates $20 \mathrm{pckts} / \mathrm{s}$. Eighteen nodes are placed at regular intervals $(1.58 \mathrm{~m})$ along a straight line without obstacles. Surrounding objects are static, with minimal timevarying changes in the wireless channel due to multipath fading effects. Fig. 2 shows the average end-to-end delays and Packet Reception Rate (PRR). The number of clusters is set depending on the channel condition and to reduce the transmission power. Both the average endto-end delays and PRR depend on the number of hops. To save energy, the RP increases the number of clusters in case of high PRR, and vice versa.

\subsection{Physical system and control setup}

Consider the T-shape ECP inverted pendulum presented in [14], which is an unstable non-minimum phase system with system matrices

$$
A=\left[\begin{array}{cccc}
0 & 1 & 0 & 0 \\
0.80 & 0 & 12.56 & 0 \\
0 & 0 & 0 & 1 \\
-2.42 & 0 & -8.33 & 0
\end{array}\right], \quad B=\left[\begin{array}{c}
0 \\
4.57 \\
0 \\
0.38
\end{array}\right]
$$

The eigenvalues of $A$ are $\lambda_{1,2}=0.74 \pm 2.08 i, \lambda_{3,4}=$ $-0.74 \pm 2.08 \mathrm{i}$. The choice of an unstable open-loop plant emphasizes the efficiency of the controller to compensate for the transmission perturbations and the performance limitations.

Experimental delay measurements $\tau_{1}$ and $\tau_{2}$ are obtained by splitting the initial experimental data into two sets, one every other sample being attributed to each set (the channels are not symmetric but belong to the same
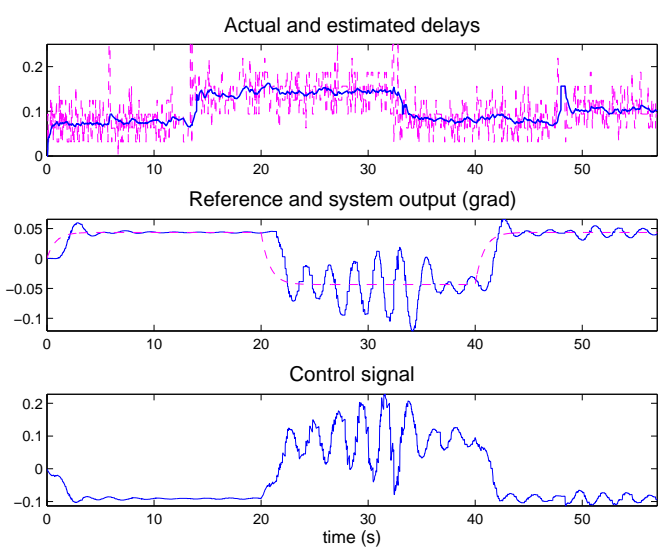

Figure 3. Delays, system output and control signal without $A R Q$.

network). Only the last received packets are considered and the packet losses are handled as described in (3). The bandwidth limitation is set by zero-order holds with a sampling time of $0.05 \mathrm{~s}$. The controller includes the time-delay estimator (based on end-to-end delay measurements), a reference trajectory block that sets the control objective, and the predictive control setup. The CUMSUM Kalman filter is set with $R=10000, Q=1$, $v=0.01$ and $g_{t h r}=35$. The reference trajectory, for the pendulum angle $x(3)$, is a filtered square signal. The predictive control is set with $\lambda=10, a=b=100$ (the introduced controller dynamics is equivalent to a lowpass filter) and $K=\left[\begin{array}{lll}0.48 & 0.362 .20 & 0.57\end{array}\right]$ sets the poles of (5) to $(-3+0.1 i ;-3-0.1 i ;-4 ;-4)$.

\subsection{Simulation results}

Fig. 3 present the actual and estimated time-delays (top), the reference and the system output (middle), and the control output (bottom). The transmissions are very noisy, in terms of jitter and packet losses (up to $60 \%$ of the signal). This figure illustrates the control scheme efficiency and the proper handling of the high frequency noise induced by the numerical integration problem. The effect of the number of clusters on the system response is particularly significant when a change in the reference sign occurs when there are three clusters. Indeed Fig. 3 depicts a situation where the bad quality of the communication channel introduces some oscillations, which are reduced as soon as the number of clusters is set back to one. Comparing the different simulation results and extra tests carried with ARQ, it appears that the system oscillations are induced by the low PRR rather than high end-to-end delay (i.e., comparing the 
different 3-clusters cases). This is due to the fact that the control setup explicitly compensate the delay effects while the robustness with respect to packet losses is not specifically addressed in the feedback design.

These simulations also show that an increase in the number of clusters has few effects on the system response when it is established in a steady state. A control oriented multi-hop wireless network protocol could efficiently use this information to minimize the emission energy (increase the number of clusters) according to some measurements of the physical state while ensuring satisfying performances.

\section{Conclusions}

We addressed the problem of remote control over a multi-hop wireless network. The constraints of such a network on the communication channel, such as timevarying delays, jitter, packet losses and bandwidth limitations are considered. The time-delay induced by the network is estimated thanks to a CUMSUM Kalman filter, that provides for a fast tracking of the mean and good suppression of the high frequency variations of the delay (jitter). This particular Kalman filter is used to design a dynamic predictive control law that compensates for the estimated part of the delay explicitly. The causality of this controller is set according to the estimator and specific considerations on safe implementation are detailed. A necessary condition on the assigned closed-loop spectrum is provided but the particular poles location is not specified, which provides for a degree of freedom for optimal control approaches and future works. Based on experimental delay and packet loss data, some simulations illustrate the proposed control scheme.

\section{References}

[1] A. Bonivento, C. Fischione, and A. SangiovanniVincentelli, "Randomized protocol stack for ubiquitous networks in indoor environment," IEEE CCNC, vol. 1, pp. 152-156, 2006.

[2] A. Lelevé, P. Fraisse, and P. Dauchez, "Telerobotics over IP networks: towards a low-level real-time architecture," in Proc. of the IEEE Int. Conf. on Intelligent Robots and Systems, vol. 2, Hawai, USA, 2001, pp. 643-648.

[3] E. Witrant, C. Canudas-de-Wit, D. Georges, and M. Alamir, "On the use of state predictors in networked control systems," in Applications of Time-Delay Systems, ser. Lecture Notes in Control and Information Sciences, J. Chiasson and J.-J. Loiseau, Eds. Springer, 2007, vol. 352.

[4] N. J. Ploplys, P. A. Kawka, and A. G. Alleyne, "Closedloop control over wireles networks," IEEE Control Syst.
Mag., vol. 24, no. 3, pp. 58-71, 2004.

[5] K. Jacobsson, N. Möller, K. H. Johansson, and H. Hjalmarsson, "Some modeling and estimation issues in traffic control of heterogeneous networks," in Proc. of the $16^{\text {th }}$ Int. Symp. on Math. Th. of Net. and Syst., Leuven, Belgium, Jul. 2004.

[6] A. Seuret, M. Termens-Ballester, A. Toguyeni, S. E. Khattabi, and J.-P. Richard, "Implementation of an internet-controlled system under variable delays," in Proc. of the IEEE Conf. on Emerging Technologies and Factory Automation, Sep. 2006, pp. 675-680.

[7] J. V. Greuen, D. Petrović, A. Bonivento, J. Rabaey, K. Ramchandran, and A. Sangiovanni-Vincentelli, "Adaptive sleep discipline for energy conservation and robustness in dense sensor networks," IEEE ICC, vol. 1, 2004.

[8] Y. Xu, J. Heidemann, and D. Estrin, "Geographyinformed energy conservation for ad hoc routing," MobiCom 2001, vol. 1, pp. 70-84, 2001.

[9] B. Chen, K. Jamieson, H. Balakrishnan, and R. Morris, "Span: An energy-efficient coordination algorithm for topology maintenance in ad hoc wireless networks," MobiCom, vol. 1, 2001.

[10] M. Kim and B. D. Noble, "Mobile network estimation." in MOBICOM, 2001, pp. 298-309.

[11] F. Gustafsson, Adaptive Filtering and Change Detection. Wiley, 2000.

[12] A. W. Olbrot, "On controllability of linear systems with time delays in the control," IEEE Transactions on Automatic Control, vol. ac-16, pp. 664-666, 1972.

[13] J.-P. Richard, "Time delay systems: An overview of some recent advances and open problems," Automatica, vol. 39, no. 10, pp. 1667-1694, Oct. 2003.

[14] E. Witrant, C. Canudas-de-Wit, D. Georges, and M. Alamir, "Remote stabilization via time-varying communication network delays: Application to TCP networks," in Proc. of the IEEE Conference on Control Applications, Taipei, Taiwan, sep 2004.

[15] S. Mondié and W. Michiels, "Finite spectrum assignment of unstable time-delay systems with a safe implementation," IEEE Transactions on Automatic Control, vol. 48, no. 12, pp. 2207-2212, Dec. 2003.

[16] P. G. Park, "Randomized protocol design in wireless automation," M. Eng. thesis, Royal Institute of Technology (KTH), Stockholm, Sweden, Jan. 2007.

[17] Tmote Sky Data Sheet, Moteiv, San Francisco, CA, 2006. [Online]. Available: http://www.moteiv.com/ products/docs/tmote-sky-datasheet.pdf 